\title{
Compact pentaquark states
}

\author{
A. Giachino ${ }^{1, *}$ and E. Santopinto ${ }^{1, * *}$ \\ ${ }^{1}$ INFN Sezione di Genova, via Dodecaneso 33, 16164 Genova, Italy
}

\begin{abstract}
We investigated the lightest pentaquark state observed by the LHCb collaboration as a compact five-quark ground- state, with spin $S=3 / 2$, and we showed that this state belongs to a $S U_{f}(3)$ flavor octet by means of a group theory approach. Moreover, we predicted in a parameter-free way the pentaquark state mass spectra for all the octet-pentaquark states. Finally, by means of an effective Lagrangian approach, we computed the octet-pentaquark partial decay widths in $J / \Psi \Lambda, J / \Psi \Sigma$ and $J / \Psi \Xi$ channels.
\end{abstract}

\section{Introduction}

In 2015, the Large Hadron Collider beauty experiment (LHCb) collaboration observed two hidden-charm pentaquarks, $P_{c}^{+}$(4380) and $P_{c}^{+}(4450)$ in $\Lambda_{b} \rightarrow J / \Psi K^{-} p$ decay [1]. This decay can proceed both by intermediate $\Lambda^{*} \rightarrow K^{-} p$ resonances, expected to be dominant, and by intermediate exotic structures, which were referred to as charmonium pentaquark states by LHCb [1]. Fig. 1 shows the number of events observed in the $J / \Psi p$ invariant mass spectrum. The data show the presence of a wider structure with an average mass value of about 4380

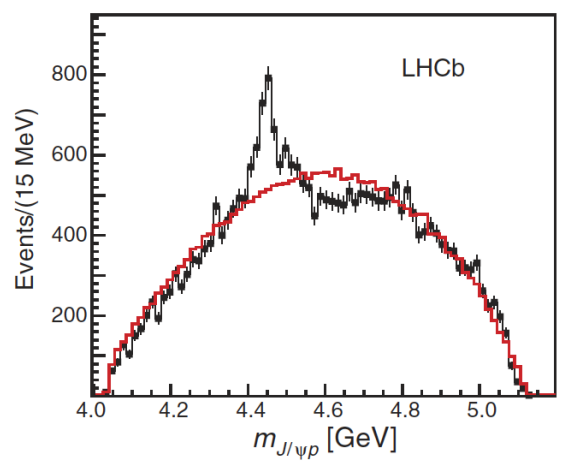

Figure 1. Number of events vs. the $J / \Psi p$ invariant mass spectrum. The red curve is the expectation from phase space. Figure adapted from ref. [1].

$\mathrm{MeV}$, called $P_{c}^{+}(4380)$ and a narrower one, with an average mass value of about $4450 \mathrm{MeV}$, called $P_{c}^{+}(4450)$. These two pentaquark states are found to have masses of $4380 \pm 8 \pm 28$

\footnotetext{
*e-mail: agiachino@ge.infn.it

**e-mail: Elena.Santopinto@ge.infn.it
} 
$\mathrm{MeV}$ and $4449.8 \pm 1.7 \pm 2.5 \mathrm{MeV}$, with corresponding widths of $205 \pm 18 \pm 86 \mathrm{MeV}$ and $39 \pm 5 \pm 19 \mathrm{MeV}$. The parities of these states are preferred to be opposite, and one state has $J=3 / 2$ and the other $J=5 / 2$.

The earliest prediction of a charmonium pentaquark state with $J^{\mathrm{P}}=\frac{3}{2}^{-}$was provided by J. J. Wu et al. [2]. Recently, Karliner e Rosner in Ref. [3] explained the heaviest pentaquark state, $P_{c}^{+}(4450)$ as a meson baryon molecular state, however the $P_{c}^{+}(4380)$ is missing in their description. For this reason, we focused on the lightest pentaquark structure by investigating it as a compact five quark state [4]. From the measured quantum numbers of the lightest resonant state, we show that it belongs to a $S U_{f}(3)$ octet with spin $S=\frac{3}{2}$. We also studied all the charmonium pentaquark states which belong to the octet, predicted their masses, and suggested possible decay channels in which the experimentalists can observe them. Recently, Wang et al. [5] calculated the pentaquark states cross section in $J / \Psi$ photoproduction and compared it with the present experimental data [6-8]. As a result, to be consistent also with $J / \Psi$ photoproduction data, they found an upper limit for branching ratio $\mathcal{B}\left(P_{c}^{+} \rightarrow J / \Psi p\right)$. By using this upper limit and our predicted masses, we calculated the partial decay widths for the predicted pentaquark resonances [4].

\section{Pentaquark mass spectra}

We think the charmonium pentaquark wave function as $q q q c \bar{c}$ where $q=u, d, s$ is a light quark and $c$ is the heavy charm quark. We adopt for each representation the notation $[f]_{d}=$ $\left[f_{1}, \ldots, f_{n}\right]_{d}$, where $f_{i}$ denotes the number of boxes in the $i$-th row of the Young tableau, and $d$ is the dimension of the representation. From a group theory based approach we found that the allowed $S U_{f}(3)$ flavour representations to which the charmonium pentaquark states can belong are an octet and a decuplet [4]:

$$
[21]_{8},[3]_{10}
$$

In order to determine what is the the lowest mass multiplet, we performed the following extension of the Gürsey-Radicati (GR) mass formula [9]:

$$
M_{G R}=M_{0}+A S(S+1)+D Y+E\left[I(I+1)-\frac{1}{4} Y^{2}\right]+G C_{2}(S U(3))+F N_{C}
$$

where $M_{0}$ is a scale parameter, $I$ and $Y$ are the isospin and hypercharge, respectively, while $C_{2}(S U(3))$ is the eigenvalue of the $S U_{f}(3)$ Casimir operator. Finally, $N_{C}$ is a counter that takes into account the mass difference between a $c$ quark (or a $\bar{c}$ antiquark) and the light quarks $(u, d)$. The coefficients in the Gürsey-Radicati (GR) mass formula extension, $A, D, E, G, F$ and the scale parameter $M_{0}$ were fitted to reproduce the spectrum of all the ground-state charmed baryons, the ground-state hyperons, and the ground-state non-strange baryons. We observe that this implies assuming that the coefficients in the GR formula are the same for different quark systems. The fitted parameters with their corresponding uncertainties are reported in Tab. 1.

According to the mass formula of Eq. 2 the lowest mass charmonium pentaquark state is the $[21]_{8} S U_{f}(3)$ octet. Therefore, in this octet, we expect to find the pentaquark state observed. As one can see form Fig. 2, the charged state $P^{0+}(4377)$ has the same quantum numbers as the lightest resonance (charge, spin, parity) reported by the LHCb. Its predicted mass is $\mathrm{M}=4377 \pm 49 \mathrm{MeV}$, in agreement with the experimental mass, $4380 \pm 8 \pm 28 \mathrm{MeV}$ $[1]$. 
Table 1. values of the parameters in the GR mass formula extension (Eq. 2) with the corresponding uncertainties.

\begin{tabular}{ccccccc}
\hline \hline & $M_{0}$ & $A$ & $D$ & $E$ & $F$ & $G$ \\
& & & & & & \\
\hline & & & & & & \\
values (MeV) & 940,0 & 23,0 & $-158,3$ & 32,0 & 1354.6 & 52,5 \\
uncertainties (MeV) & 1.5 & 1.2 & 1.3 & 1.3 & 18.2 & 1.3 \\
\hline \hline
\end{tabular}

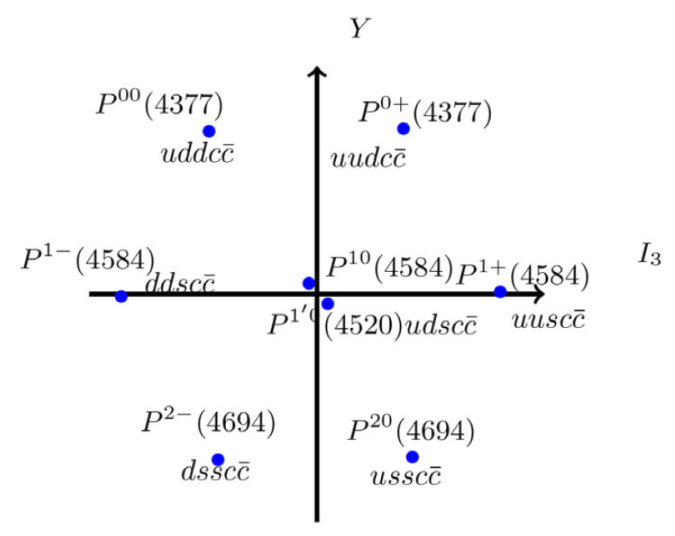

Figure 2. Octet of the charmonium pentaquark states. A pentaquark state is denoted with $P^{i j}(M)$, where $i=0,1,2$ is the number of strange quarks, $j=-, 0,+$ is the pentaquark's electric charge, and $M$ the predicted mass obtained with Eq. 2.

\section{Pentaquark partial decay widths}

In calculating the decay widths of the predicted pentaquark states, reported in Fig. 2, we adopted an effective Lagrangian proposed in Ref. [10] for $p N J / \psi$ coupling. Wang et al. [5] calculated the cross-section of the pentaquark states in $J / \Psi$ photoproduction and compared it with the available experimental data ([6-8]). He noticed also that, since the pentaquark states have been observed in $J / \Psi p$ channel, it should be produced also in $J / \Psi p$ photoproduction [5]. This gives a constraint for the coupling strength between $J / \Psi p$ and the two pentaquark states. In fact, in order to have consistency with the available photoproduction data the branching ratio upper limit for both the pentaquark states is $\mathcal{B}\left(P^{+} \rightarrow J / \Psi p\right) \leq 0.05$. From this constraint and the effective lagrangian proposed by S. H. Kim in [10] we calculated the $J / \Psi p$ coupling constant, $\bar{g}_{p J / \Psi}=\frac{g_{1}}{2 M_{N}}$. Moreover, from our pentaquark mass predictions we were also able to calculate the partial decay widths for the pentaquark states with open strangeness. For example, the $P^{1+}$ partial decay width in the $\Sigma^{+} J / \Psi$ channel, $\Gamma\left(P^{1+} \rightarrow \Sigma^{+} J / \psi\right)$, is given by:

$\Gamma\left(P^{1+} \rightarrow \Sigma^{+} J / \psi\right)=\frac{\bar{g}_{\Sigma^{+} J / \Psi}^{2}}{12 \pi} \frac{p_{\Sigma^{+}}}{M_{P^{1^{\prime}}}}\left(E_{\Sigma^{+}}+M_{\Sigma^{+}}\right)\left[2 E_{\Sigma^{+}}\left(E_{\Sigma^{+}}-M_{\Sigma^{+}}\right)+\left(M_{P^{1+}}-M_{\Sigma^{+}}\right)^{2}+2 M_{J / \psi}^{2}\right]$ 
where kinematic variables are related by the relativistic equation $p_{\Sigma^{+}}=\sqrt{E_{\Sigma^{+}}^{2}-M_{\Sigma^{+}}^{2}}$, and the coupling constant $\bar{g}_{\Sigma^{+} J / \Psi}$, is related to the $J / \Psi p$ coupling constant by the following rescaling:

$$
\bar{g}_{\Sigma^{+} J / \Psi}=\frac{g_{1}}{2 M_{\Sigma^{+}}} .
$$

If we use the upper branching ratio limit extracted by Wang [5], $\mathcal{B}\left(P^{+} \rightarrow J / \Psi p\right)=0.05$, and the total width reported by LHCb, $\Gamma_{t o t}=205 \mathrm{MeV}$, we obtain for the $P_{c}^{+}(4380)$ partial decay width in $J / \psi p$ channel:

$$
\Gamma_{N J / \Psi}=\mathcal{B}\left(P^{+} \rightarrow J / \Psi p\right) \Gamma_{t o t}=10.25 \mathrm{MeV} .
$$

The numerical results for the other channels are listed in Table 2.

Table 2. Partial decay widths for $\Lambda J / \Psi, \Sigma J / \Psi$ and $\Xi J / \Psi$ channels, calculated from the constraint that $J / \Psi p$ channel accounts for the $5 \%$ of the total pentaquark width, according to Ref. [5]. The notation used to denote a pentaquark state is the same as the one introduced in 2 .

\begin{tabular}{ccc}
\hline \hline initial state & channel & $\begin{array}{c}\text { partial } \\
\text { width }(\mathrm{MeV})\end{array}$ \\
\hline$P^{1^{\prime} 0}$ & $\Lambda J / \Psi$ & 7.94 \\
$P^{1-}, P^{10}, P^{1+}$ & $\Sigma J / \Psi$ & 7.21 \\
$P^{2-}, P^{20}$ & $\Xi J / \Psi$ & 6.35 \\
\hline
\end{tabular}

\section{Conclusions}

We have studied all the charmonium pentaquark states which belong to the flavour octet, predicted their masses, and suggested possible bottom baryon decay channels which involve the predicted resonances as intermediate states. Finally, we computed the partial decay widths for all the suggested octet-pentaquark decay channels.

\section{References}

[1] R. Aaij et al. [LHCb Collaboration], Phys. Rev. Lett. 115, 072001 (2015).

[2] J. J. Wu et al., Phys. Rev. Lett. 105, 232001 (2010)

[3] M. Karliner and J. L. Rosner, Phys. Rev. Lett. 115, 122001 (2015).

[4] E.Santopinto and A Giachino, Phys.Rev. D 96, 014014 (2017).

[5] Q. Wang, X. H. Liu, and Q. Zhao, Phys. Rev. D 92, 034022 (2015).

[6] U. Camerini et al., Phys. Rev. Lett. 35, 483 (1975).

[7] R. L. Anderson, Report No. SLAC-PUB-1417.

[8] B. Gittelman et al., Phys. Rev. Lett. 35, 1616 (1975).

[9] F. Gursey and L.A. Radicati, Phys. Rev. Lett. 13, 173 (1964)

[10] S. H. Kim, S. i. Nam, Y. Oh, and H.-Ch. Kim, Phys. Rev. D 84, 114023 (2011). 